\title{
Validez y fiabilidad de la versión española de la escala 'perfil de deterioro cognitivo grave' (SCIP) para la evaluación cognitiva de personas con enfermedad de Alzheimer en estadios avanzados
}

\author{
Cristina Buiza, Ignacio Montorio-Cerrato, Javier Yanguas
}

Introducción. Existen pocas herramientas validadas al castellano para realizar una evaluación precisa del funcionamiento
cognitivo de personas con demencias avanzadas. Esta población supone un reto por el elevado número de personas que
alcanzan estos estadios y por la complejidad de cuidados que requieren. Este trabajo presenta la validación al castellano
de un instrumento, la escala 'perfil de deterioro cognitivo grave' (SCIP), que permite la evaluación del funcionamiento
cognitivo de personas con enfermedad de Alzheimer avanzada.

Pacientes y métodos. Estudio transversal con 133 sujetos (29 hombres y 104 mujeres; edad media: 81,61 \pm 7,41 años) con diagnóstico de enfermedad de Alzheimer en estadios de moderado a muy grave, según la escala de deterioro global (GDS 5-7). Se realizaron análisis de discriminación de los ítems, estudio de la consistencia interna y fiabilidad interjueces y testretest, y se contrastó la estructura factorial y la validez del instrumento.

Resultados. Sólo el 1\% de los 160 ítems de la escala resultó no discriminativo. La consistencia interna de la escala es adecuada, así como la fiabilidad interjueces y test-retest. Respecto a la validez de constructo, la correlación con el miniexamen cognitivo es $0,74(p<0,01)$, y se encuentran diferencias significativas entre la escala SCIP y los tres niveles de la GDS analizados. La estructura factorial de la escala muestra la existencia de un solo factor que explica el 66,12\% de la varianza.

Conclusión. La escala SCIP es un instrumento útil, fiable y válido para la evaluación exhaustiva en castellano de las funciones cognitivas en personas con demencia en estadios de moderado a muy grave.

Palabras clave. Demencia avanzada. Deterioro cognitivo grave. Enfermedad de Alzheimer. Evaluación neuropsicológica. Perfil de deterioro cognitivo grave. SCIP. Validación.

\section{Introducción}

Diferentes estudios señalan que la demencia avanzada supone un gran reto sociosanitario en los países desarrollados [1,2], y que una evaluación precisa del estado cognitivo de estas personas es una necesidad que hay que resolver si se pretende ofrecer un cuidado adecuado a sus características de funcionamiento [3-6].

La mayoría de los tests usados para evaluar la demencia en grados leve y moderado tiene una sensibilidad limitada para la valoración de los pacientes más deteriorados, en parte provocada por el deterioro en las habilidades de lenguaje que existe en esas fases de la demencia, y que hace que el paciente sea incapaz de comprender qué tarea se le pide que realice en la prueba [7]. En estas pruebas, es común encontrarse con lo que se ha llamado 'efecto suelo', es decir, las personas evaluadas puntúan en el límite inferior de la prueba, ya que el nivel reque- rido para realizarla es demasiado alto para sus capacidades, lo que no permite diferenciar niveles de ejecución inferiores [7].

La experiencia clínica sugiere que los pacientes gravemente deteriorados pueden mostrar un amplio rango de funcionamiento en las capacidades cognitivas básicas (por ejemplo, comportamiento social no verbal, tareas de clasificación simples o identificación de colores) a pesar de tener graves déficits cognitivos en otras áreas, lo que pone en duda el punto de vista tradicional de que estos pacientes están deteriorados de forma profunda y homogénea en todas las funciones [8].

Sin embargo, sólo existen hasta la fecha dos instrumentos validados en castellano para la evaluación de personas con este perfil cognitivo: la batería de deterioro grave (SIB) [9], así como su versión abreviada (SIB-s) [10], y el miniexamen del estado mental grave (SMMSE) [11]. La primera de las pruebas ofrece buenas propiedades psicométricas y el tipo
Facultad de Psicología; Universidad Autónoma de Madrid; Madrid (I. Montorio-Cerrato. Centro Gerontológico Julián Rezola; Matia Fundazioa (C. Buiza). Matia Instituto Gerontológico (J. Yanguas). San Sebastián, España.

Correspondencia:

Dra. Cristina Buiza Bueno. Centro Gerontológico Julián Rezola. Matia Fundazioa. Avda. Zarautz, 123. E-20018 San Sebastián

E-mail:

buizabueno@gmail.com

Aceptado tras revisión externa: 14.09.15.

Cómo citar este artículo: Buiza C, Montorio-Cerrato I, Yanguas J. Validez y fiabilidad de la versión española de la escala 'perfil de deterioro cognitivo grave' (SCIP) para la evaluación cognitiva de personas con enfermedad de Alzheimer en estadios avanzados. Rev Neurol 2015; 61: 349-56.

(c) 2015 Revista de Neurología 
de tareas con las que evalúa son adecuadas para sujetos con demencia avanzada, pero ofrece una gran carga de memoria explícita, lo que dificulta la exploración en sujetos con deterioro muy avanzado. Además, aunque ofrece una puntuación diferenciada por áreas de funcionamiento cognitivo, no ayuda a establecer subgrupos de gravedad de deterioro. En el caso del SMMSE, se trata de una prueba breve de medida del estado cognitivo general, posee también buenas características psicométricas, y resulta un instrumento fácil y rápido de aplicar; sin embargo, no ofrece un perfil de funcionamiento cognitivo detallado.

La prueba seleccionada para este estudio, la escala 'perfil de deterioro cognitivo grave' -Severe Cognitive Impairment Profile (SCIP) - [12], permite la obtención de un perfil de ejecución en cada una de las dimensiones cognitivas evaluadas y el cálculo de una puntuación total indicativa del grado de deterioro cognitivo. El rango de esta puntuación oscila entre 0 y 245 puntos, lo que permite que la SCIP sea suficientemente sensible para discriminar dentro de un amplio rango de funcionamiento cognitivo. Además, una de las ventajas adicionales de la SCIP es que propone cuatro grupos de división del deterioro, según la puntuación total de la escala: moderadamente grave, grave, muy grave y profundo, diferenciación que resulta útil en la clasificación clínica de personas en estadios avanzados de la demencia y se aleja de considerar a este grupo de personas como un todo. La SCIP [12] se desarrolló con el fin de ofrecer información detallada sobre el funcionamiento en un amplio rango de funciones cognitivas en pacientes con demencia avanzada, con una adecuada representación de diferentes niveles de dificultad en cada una de las funciones. Cubre ocho áreas del funcionamiento cognitivo: conducta social, atención, lenguaje, memoria, aritmética, funcionamiento motor, conceptualización y funcionamiento visuoespacial. Todos los ítems se administran y se puntúan según unas instrucciones estándares, que tienen en cuenta las dificultades que suelen presentarse en la evaluación de participantes con un deterioro cognitivo avanzado (por ejemplo, déficits graves de atención). En el estudio de validación original de la escala, ésta presenta una fiabilidad test-retest e interjueces altas (coeficientes de correlación test-retest para las subescalas y la prueba total entre 0,56 y 0,96 ; coeficientes de correlación interjueces para las subescalas y la prueba total entre 0,77 y 1 ), y una buena validez de constructo, con una alta correlación entre las puntuaciones de la SCIP y las de la escala de estadificación de la demencia de Mattis [13] $(r=0,91 ; p<0,001)$, entre la SCIP y el MMSE [14] $(r=0,84 ; p<0,001)$, y entre la SCIP y la SIB [8] $(r=0,93 ; p<0,001)$. En el estudio de validación de la escala, los autores proponen cuatro subgrupos de división del deterioro, cuyos puntos de corte en la escala serían: deterioro moderadamente grave, $>198$; deterioro grave, 131-198; deterioro muy grave, 72-130; y deterioro profundo, $<72$ [12]. Las principales debilidades de la escala se deben a los problemas en la cantidad, homogeneidad y tratamiento estadístico de las normas en su proceso de validación [15].

El objetivo principal del estudio ha sido realizar la adaptación y validación al castellano de la SCIP [12], estudiando su aplicabilidad, así como sus principales propiedades psicométricas.

\section{Pacientes y métodos}

\section{Muestra}

Se desarrolló un estudio transversal a partir de una muestra de 133 pacientes, que cumplían con los siguientes criterios de inclusión:

- Diagnóstico de enfermedad de Alzheimer probable, según los criterios NINCDS-ADRDA [16], en estadios de moderado a muy grave.

- Puntuación en la escala de deterioro global (GDS) $\geq 5$ [17].

- Haber firmado el consentimiento informado para la participación en el estudio por parte de un familiar o responsable/tutor.

Se excluyó del estudio a las personas que presentaran otros diagnósticos de origen neurológico, historia de enfermedad psiquiátrica, historia de abuso de alcohol $\mathrm{u}$ otras sustancias, que estuvieran encamadas o que presentaran otras enfermedades que redujeran su esperanza de vida a menos de seis meses.

La muestra la formaban 29 hombres $(21,8 \%)$ y 104 mujeres $(78,2 \%)$ que en el momento de la evaluación estaban viviendo en su domicilio o en centros residenciales del País Vasco y Navarra. La edad media fue de 81,61 7,41 años (rango: 57-96 años).

\section{Aspectos éticos}

El protocolo de investigación fue aprobado por el Comité de Ética en la Investigación de Matia-Hurkoa-Gerozerlan. Todos los participantes en el estudio o sus familiares fueron informados de manera individual por el investigador principal y firmaron el consentimiento informado. Todos los datos personales fueron recogidos, almacenados y protegidos 
según las normas de la Ley Orgánica 15/1999, de 23 de diciembre, para la Protección de Datos Personales (LOPD 15/1999).

\section{Protocolo de evaluación}

La batería de pruebas administrada para la evaluación de la muestra incluye datos sociodemográficos (sexo, edad, nivel educativo y estado civil), pruebas de la GDS [17] y de funcionamiento cognitivo - miniexamen cognitivo (MEC) [18] y SCIP [12]-. Las pruebas administradas se describen a continuación.

\section{Estadificación del deterioro global}

La GDS [17] se basa en un esquema de progresión del deterioro generalizado y jerárquico, que describe siete estadios de demencia, desde la normalidad (estadio 1) al deterioro grave (estadio 7). Asume un deterioro progresivo a través de todas las funciones cognitivas basado en el deterioro que se produce en la enfermedad de Alzheimer, un hecho que a veces hace que sea difícil de aplicar a pacientes con otro tipo de demencias, aunque se trata de una escala clínica ampliamente utilizada internacionalmente.

\section{Estado cognitivo}

El MEC [18] es el test breve de cribado del deterioro cognitivo más difundido en la clínica y en la investigación. Fue diseñado basándose en el MMSE creado por Folstein et al [14]. El MEC es la primera versión en castellano, adaptada y validada por Lobo et al $[18,19]$. Se trata de un instrumento que consta de 24 ítems que exploran cinco áreas cognitivas: orientación, fijación, concentración y cálculo, memoria y lenguaje. La puntuación total máxima es de 35 puntos. El MEC ha demostrado en diferentes estudios su fiabilidad, validez y poder discriminativo.

Respecto a la SCIP [12], en el presente estudio se realizó el proceso de traducción-retrotraducción de la escala original. La figura ofrece un esquema del proceso que se describe a continuación. La escala original fue traducida al castellano buscando una traducción semántica y no literal para asegurar la equivalencia conceptual e idiomática entre ambas versiones. Esa traducción fue revisada por un grupo de expertos y traducida de nuevo al inglés por dos traductores independientes. El grupo de expertos volvió a analizar las dos versiones y su equivalencia con la versión original, y los ítems que presentaban problemas de interpretación se discutieron y se llegó a un acuerdo sobre una primera versión piloto en castellano que fue aplicada a una pequeña muestra para comprobar su aplicabilidad y comprensión. Los resultados de este estudio de apli-
Figura. Esquema del proceso de traducción-retrotraducción y adaptación de la escala 'perfil de deterioro cognitivo grave'.

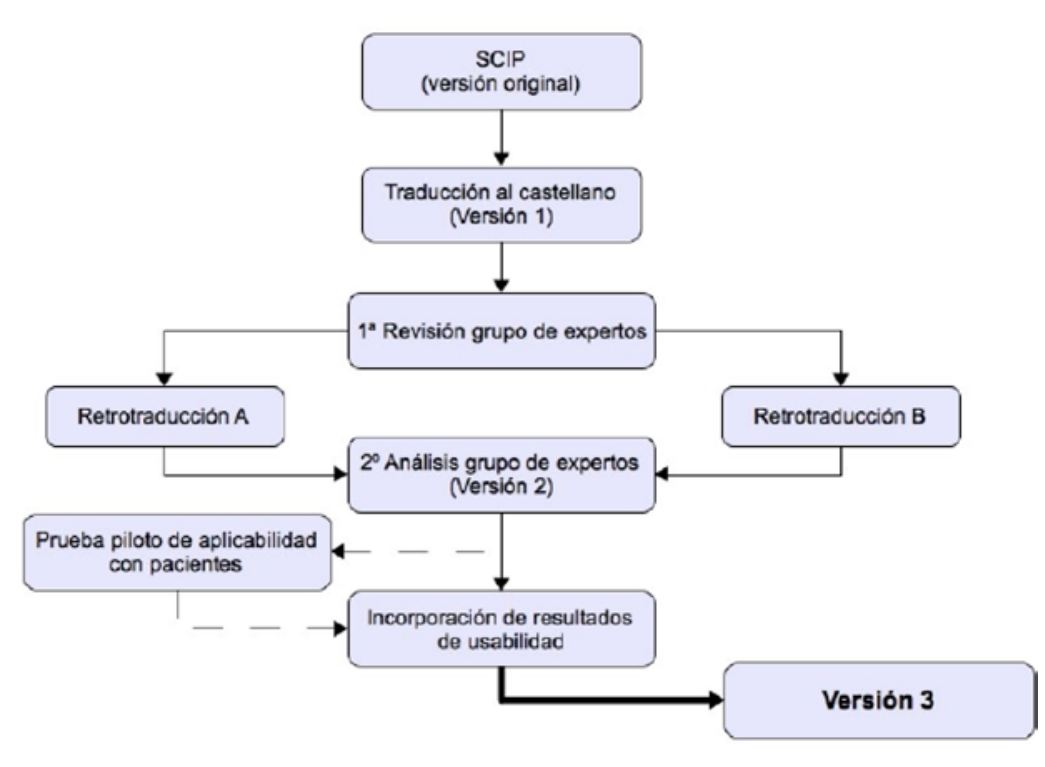

cabilidad se incorporaron a la prueba, lo que permitió elaborar la versión definitiva utilizada en este estudio. Para una información más detallada del proceso de adaptación de la prueba al castellano se puede consultar el trabajo original de Buiza [7].

\section{Análisis estadístico}

Una vez efectuada la recogida de datos, se procedió a su codificación. Tras la depuración inicial de los datos y un análisis de la comprobación de la precisión conseguida en la introducción de los datos (porcentaje de error inferior a 0,04 tras revisar el $10 \%$ del total), se procedió a su análisis estadístico, que se efectuó utilizando versiones sucesivas del programa SPSS.

En primer lugar, se realizaron análisis descriptivos de los participantes que muestran datos normativos y de distribución de las puntuaciones de los participantes en las variables estudiadas. Posteriormente, se analizó la capacidad de discriminación de los ítems y de las subescalas que componen la prueba. Se realizaron análisis de consistencia interna mediante el coeficiente $\alpha$ de Cronbach. El análisis de fiabilidad interjueces se realizó mediante el cálculo del coeficiente de correlación intraclase, y la fiabilidad test-retest, mediante el coeficiente de correlación de Pearson. En el caso de la fiabilidad interjueces, se realizaron coeficientes de correlación entre 
Tabla I. Coeficientes de consistencia interna, fiabilidad interjueces y fiabilidad test-retest de cada subescala de la escala 'perfil de deterioro cognitivo grave'.

\begin{tabular}{lccc}
\hline & $\begin{array}{c}\text { Consistencia } \\
\text { interna }(\alpha)\end{array}$ & $\begin{array}{c}\text { Fiabilidad } \\
\text { interjueces (ICC) }\end{array}$ & $\begin{array}{c}\text { Fiabilidad } \\
\text { test-retest }(r)\end{array}$ \\
\hline Conducta social & 0,91 & $0,86^{a}$ & $0,98^{a}$ \\
\hline Atención & 0,69 & $0,99^{a}$ & $0,93^{a}$ \\
\hline Lenguaje & 0,76 & $0,99^{a}$ & $0,97^{a}$ \\
\hline Memoria & 0,83 & $0,99^{a}$ & $0,91^{a}$ \\
\hline Motor & 0,07 & $1,00^{a}$ & $1,00^{a}$ \\
\hline Conceptualización & 0,69 & $0,97^{a}$ & $0,98^{a}$ \\
\hline Aritmética & 0,84 & $0,98^{a}$ & $0,85^{a}$ \\
\hline Visuoespacial & 0,87 & $0,93^{a}$ & $0,91^{a}$ \\
\hline a $p<0,01$. & & & \\
\hline
\end{tabular}

las puntuaciones obtenidas por dos jueces independientes puntuando al mismo sujeto simultáneamente. Para la fiabilidad test-retest, se realizaron coeficientes de correlación entre las puntuaciones obtenidas por un mismo sujeto en dos mediciones, con una diferencia de una semana entre ellas. Mediante el cálculo del coeficiente de correlación de Pearson, se analizaron las relaciones entre las puntuaciones totales de la SCIP y las puntuaciones totales del MEC para determinar la validez de constructo. La validez convergente se estableció mediante un análisis de varianza de un factor (ANOVA), tomando como factor cada uno de los tres estadios de enfermedad de Alzheimer estudiados (GDS 5, 6 y 7), y como variable criterio, la puntuación obtenida en la SCIP. Asimismo, se compararon las frecuencias de la distribución de participantes entre las tres categorías GDS y las cuatro establecidas por la SCIP mediante el cálculo del coeficiente de contingencia. Por último, para determinar la estructura factorial de la escala, se realizó un análisis de componentes principales sobre las ocho subescalas que componen la prueba.

\section{Resultados}

\section{Descripción de la muestra}

La mayor parte de la muestra $(66,2 \%)$ se situaba en un nivel 5 de estadificación según la GDS, y el resto de los participantes, en el GDS $6(27,8 \%)$ o GDS 7 (6\%). La media obtenida en el MEC y en la SCIP fue de $11,8 \pm 6,38$ y $175,7 \pm 55,81$, respectivamente.

\section{Capacidad de discriminación}

Se analizaron las frecuencias de respuesta que dieron los participantes de la muestra a cada uno de los ítems y escalas. Se consideró que un ítem no es capaz de discriminar y, por lo tanto, podría eliminarse de la prueba cuando el $95 \%$ o más de las respuestas de los participantes se acumulan en una única categoría de respuesta. Sólo tres de los 160 ítems que componen la prueba cumplían ese criterio, por lo que se podían considerar incapaces de discriminar.

\section{Fiabilidad}

Se estableció una consistencia interna total para la prueba de 0,85 . Por subescalas, se obtuvieron unos coeficientes de consistencia interna que oscilaron entre 0,69 (subescala de atención) y 0,91 (subescala de comportamiento).

Para la fiabilidad interjueces, el coeficiente de correlación intraclase hallado entre los resultados de ambas evaluaciones fue significativo para la puntuación total (coeficiente de correlación intraclase $=0,87 ; p<0,01$ ). La fiabilidad interjueces asegura que la codificación de la respuesta del sujeto no está influida por la posible interpretación que de ésta haga el evaluador. Los resultados de los análisis de fiabilidad test-retest determinaron que fueron significativos para la puntuación global $(r=0,99 ; p<$ 0,01 ), así como para las ocho subescalas, con coeficientes que variaban entre 0,85 y 1 . En la tabla I se muestran los resultados de los análisis de fiabilidad realizados para cada una de las ocho subescalas que la componen.

\section{Validez de constructo}

Para el estudio de la validez de constructo se compararon las puntuaciones en la escala con dos medidas ampliamente utilizadas de funcionamiento cognitivo, como el MEC y la GDS. Las puntuaciones obtenidas en la escala total de la SCIP mostraron una correlación con la puntuación total del MEC de $0,74(p<0,01)$.

En el análisis de varianza de un factor (ANOVA) realizado con las medias obtenidas en la puntuación total en la escala de cada uno de los tres estadios de enfermedad de Alzheimer analizados (GDS 5, 6 y 7), se encontró que existían diferencias significativas en las puntuaciones totales en la SCIP en- 
tre los tres niveles de la GDS ( $F=93,34 ; p<0,01)$. En la tabla II se ofrecen las puntuaciones obtenidas en el análisis. El test de comparaciones múltiples reveló que las puntuaciones totales en la SCIP eran diferentes entre los tres niveles de la GDS. El coeficiente de contingencia entre las frecuencias acumuladas de las tres categorías GDS y las cuatro SCIP fue de $0,57(p<0,001)$.

Para determinar la estructura factorial de la escala, se realizó un análisis de componentes principales sobre las ocho subescalas. La adecuación de la muestra para el análisis factorial se realizó mediante la medida Kaiser-Meyer-Olkin $(\mathrm{KOM}=0,92)$, indicando que los patrones de correlaciones son muy compactos y, por lo tanto, el análisis factorial determinará factores distintivos y fiables. El test de esfericidad de Bartlett $\left(\chi_{(28)}^{2}=753,44 ; p<0,01\right)$ indicó que las correlaciones entre los ítems son suficientemente altas para la realización de un análisis de componentes principales. Utilizando el método de extracción de análisis de componentes principales, se obtuvo un solo factor que explica el $66,12 \%$ de la varianza. En la tabla III se expone la matriz de componentes del análisis factorial.

\section{Discusión}

El presente estudio muestra el proceso de adaptación de la SCIP y ofrece datos de sus propiedades psicométricas en una muestra de sujetos con diagnóstico de enfermedad de Alzheimer en estadios avanzados. Para cumplir con el objetivo de realizar la validación al castellano de la SCIP, se realizaron los análisis de discriminación de ítems, fiabilidad y validez descritos.

Se encontraron tres ítems que no eran discriminativos, pero se decidió no eliminar ninguno de ellos, ya que permiten discriminar a una muy pequeña proporción de sujetos, pero que a efectos evolutivos puede estar marcando los estadios más avanzados y de mayor deterioro; resulta interesante su mantenimiento en la escala para conservar la sensibilidad de la prueba y no crear un posible 'efecto suelo'.

Para el estudio de la fiabilidad de la versión adaptada de la prueba, se realizó un análisis de su consistencia interna, que resulta alta $(0,85)$. En la validación original de la prueba no se informó sobre los resultados de esta medida [12]. En otras escalas de evaluación del funcionamiento cognitivo para demencias avanzadas se han hallado coeficientes de consistencia interna que varían desde 0,91 [20] hasta 0,94 [21], aunque la mayor parte de las escalas
Tabla II. Diferencias en la puntuación total en la escala 'perfil de deterioro cognitivo grave' en función de los niveles de estadificación de la enfermedad de Alzheimer según la escala de deterioro global (CDS).

\begin{tabular}{|c|c|c|c|c|c|}
\hline & \multirow{2}{*}{$\begin{array}{c}\text { Media } \pm \\
\text { desviación } \\
\text { estándar }\end{array}$} & \multirow{2}{*}{$F(p)$} & \multicolumn{3}{|c|}{ Diferencias entre los grupos } \\
\hline & & & GDS 5 & GDS 6 & GDS 7 \\
\hline GDS 5 & $202,9 \pm 27,42$ & & & $p<0,01$ & $p<0,01$ \\
\hline GDS 6 & $137,9 \pm 53,19$ & & & & $p<0,01$ \\
\hline GDS 7 & $51,1 \pm 14,73$ & $93,34(0,00)$ & & & \\
\hline
\end{tabular}

Tabla III. Matriz de componentes del análisis de componentes principales de las subescalas que componen la escala 'perfil de deterioro cognitivo grave'

\begin{tabular}{lcc}
\hline & Componente 1 & $\begin{array}{c}\text { Comunalidades } \\
\text { (extraction) }\end{array}$ \\
\hline Conducta social & 0,84 & 0,70 \\
\hline Atención & 0,86 & 0,75 \\
\hline Lenguaje & 0,88 & 0,77 \\
\hline Memoria & 0,84 & 0,70 \\
\hline Funcionamiento motor & 0,56 & 0,31 \\
\hline Conceptualización & 0,88 & 0,78 \\
\hline Aritmética & 0,74 & 0,54 \\
\hline Visuoespacial & 0,86 & 0,75 \\
\hline
\end{tabular}

analizadas no ofrece información sobre la consistencia interna en sus estudios de validación. Respecto a la consistencia interna de cada una de las subescalas que componen la prueba, se encontraron coeficientes suficientemente altos para garantizar esta propiedad. No obstante, la subescala de funcionamiento motor tiene unas características de ejecución que presenta distintos problemas en su aplicación y propiedades psicométricas. Teniendo en cuenta el coeficiente $\alpha$ obtenido por la subescala motor $(0,07)$, se constata que la escala presenta problemas en su construcción y que no mide adecuadamente la habilidad motora de los sujetos con demencia avanzada. Tal subescala se aplica pidiendo al sujeto que inserte unos aros en un palo que se sitúa sobre la mesa, tarea que debe hacer en un tiempo máximo de ejecución. Debido a que el tiempo máximo permitido es muy alto, prácticamente la to- 
talidad de los sujetos se situaron en los dos extremos de medida de la subescala, ya que quien no es capaz de realizar la tarea hace 'suelo', y a los sujetos que pueden realizarla, aunque sea muy despacio, les da tiempo a completarla, por lo que hacen 'techo'. Para intentar eliminar este efecto, se realizó una ponderación en la que se penalizaba según el cuartil en el que se situaba el tiempo que se tardó en finalizar la tarea, pero tampoco mejoraron la fiabilidad ni la validez de la subescala [7]. La autora de la escala original obtuvo los mismos resultados, y lo explica basándose en que la tarea se diseñó principalmente como un cribado de funcionamiento motor simple [8].

Se realizó, además, un análisis de la fiabilidad interjueces y test-retest de la escala, y tanto la escala total como cada una de las subescalas que componen la SCIP muestran una muy buena fiabilidad interjueces, lo que indica que la escala puede ser administrada por distintos evaluadores asegurando que las puntuaciones que obtendrá el sujeto serán las mismas. Al igual que sucede en la validación original de la escala, la subescala que menor coeficiente de fiabilidad interjueces obtiene es la subescala de conducta social $(0,77$ en la original frente al 0,88 de la validación al castellano). Peavy et al explican estos resultados por la subjetividad de los ítems que componen esta escala, e indican que éstos son los más susceptibles de toda la prueba a las fluctuaciones que pueda sufrir la persona con demencia en su estado de ánimo y motivacional, lo que causa también una menor fiabilidad test-retest en esta escala [12].

Los resultados de fiabilidad test-retest pueden considerarse suficientemente altos como para afirmar que cada una de las escalas tiene una suficiente estabilidad temporal. No obstante, el estado cognitivo en una enfermedad degenerativa como la enfermedad de Alzheimer sufre variaciones debidas a la evolución de la patología con el paso del tiempo, por lo que no es de esperar una correlación perfecta entre ambas medidas temporales.

Respecto a la validez de la prueba, los resultados del análisis de componentes principales indican que la escala puede ser categorizada en un solo factor claramente identificable. Estos resultados apoyan que la SCIP es una escala con estructura unifactorial, lo que muestra la existencia de un factor subyacente que refleja una disfunción cognitiva general y refuerza su validez de constructo.

La validez convergente se estudió comparando las puntuaciones obtenidas en la SCIP con las puntuaciones obtenidas por los mismos sujetos en el MEC y la GDS. Estos dos tests fueron elegidos como estándar a partir del cual evaluar la validez de la esca- la SCIP, debido a que ambos han mostrado ser sensibles al deterioro cognitivo y ambos han sido validados en estudios con confirmación del diagnóstico por autopsia [22]. La correlación obtenida entre la SCIP y el MEC resultó alta, pero no supera el valor de 0,8. Este hecho refuerza la idea de que la SCIP posee un rango más amplio de capacidad de medición de la ejecución cognitiva, extendiendo el rango inferior de puntuación del MEC. Ambas escalas, por lo tanto, están midiendo un mismo constructo, funcionamiento cognitivo, pero en diferente rango de puntuación, y comparten un rango común, el de los estadios moderados de demencia.

Respecto a la utilización de la GDS para contrastar la validez de la SCIP, como era esperable se encuentran diferencias significativas en las puntuaciones medias de la SCIP en cada uno de los tres niveles de GDS, y son menores las puntuaciones en los niveles más altos de la GDS, indicativos de mayor pérdida funcional. Cabe preguntarse cuál es el valor añadido de contar con una escala como la SCIP frente a la tradicional clasificación de la GDS. En este sentido, la SCIP permite obtener una puntuación dimensional y continua con un amplísimo rango de respuesta frente a la distinción de sólo tres categorías desde la perspectiva de la GDS. Por otra parte, la utilización de las categorías de la SCIP frente a las categorías de la GDS también supone una ventaja. Al comparar los rangos de puntuaciones de la SCIP entre las propias categorías de la SCIP y de la GDS, se observa que una parte muy sustantiva de los participantes se encuentran en la GDS 5 (dos de cada tres), mientras que hay mayor variabilidad en la distribución de participantes en la clasificación de la SCIP, ya que la categoría con el mayor grado de participantes no llega al 50\% (46\% en la categoría de la SCIP 'grave'). Ello viene a señalar que la GDS tiene menor sensibilidad y poder discriminativo, ya que sitúa a la mayor parte de los sujetos en el mejor nivel de la GDS. Por el contrario, incluso la categoría de la SCIP indicativa de un menor nivel cognitivo, como es 'deterioro profundo', engloba a un significativo $22 \%$ de los participantes. Además, ambas escalas tienen una estrecha relación que se evidencia en que el 94\% de los participantes clasificados como 'moderadamente grave' de la SCIP pertenezcan a la GDS 5, que el 90\% de los clasificados 'profundo' se sitúen en la GDS 6 y 7, y en el propio y significativo coeficiente de contingencia entre ambas categorías, que, aunque no es muy elevado, sí muestra dependencia entre ambas escalas. La especificidad cognitiva de la SCIP frente al fuerte peso en discriminación funcional de la GDS contribuye a explicar estos resultados. 
Este estudio presenta algunas limitaciones. En primer lugar, la selección de la muestra no fue aleatoria, sino de conveniencia. Hubiese sido deseable contar con un mayor número de participantes, especialmente de aquellos con un mayor nivel de deterioro, aunque las características de ese grupo hacen más difícil su participación en este tipo de estudios. Además, la muestra estuvo formada por pacientes con diagnóstico de enfermedad de Alzheimer, y para futuros estudios convendría poder incluir pacientes con distintas etiologías de demencia. Para la realización del análisis de la validez convergente, quizá la utilización de otros instrumentos, como el SIB [8,9], hubiera resultado más adecuada. Finalmente, la medida de distintas variables en sujetos cuyas capacidades cognitivas se encuentran muy alteradas plantea un problema metodológico importante relacionado con la necesidad de que los pacientes emitan respuestas verbales. Sería importante contar con medidas precisas de funcionamiento cognitivo en demencias avanzadas que estuvieran exentas de la mediación del lenguaje.

Los resultados de este estudio permiten concluir que la SCIP es un instrumento útil, valido y fiable para su uso en la evaluación del estado cognitivo de personas con demencia avanzada. La SCIP permite eliminar el efecto suelo en la medida de las capacidades cognitivas de sujetos con demencia avanzada. Por el perfil de puntuaciones ofrecidas, permite analizar la ejecución cognitiva global o cada función cognitiva de manera individualizada. Además, la escala permite establecer cuatro subgrupos de deterioro, dentro de lo que se considera grave. Con estos resultados, lo que hasta ahora era considerado como una única categoría puede clasificarse de una manera distintiva en subgrupos de gravedad. El grupo de sujetos clasificados como demencia avanzada es muy heterogéneo y presenta unas características de funcionamiento variadas. Para ofrecer planes de atención centrados en la persona en estos sujetos, es necesario contar con herramientas precisas de evaluación que permitan el diseño adecuado de intervenciones, planes de cuidado y estrategias de comunicación eficaces.

Bibliografía

1. Martín-Sánchez FJ, Gil-Gregorio P. Valoración funcional en la demencia grave. Rev Esp Geriatr Gerontol 2006; 41 (Supl 1): S43-9.

2. López-Mongil R, López-Trigo JA, Castrodeza-Sanz FJ, Tamames-Gómez S, León-Colombo T. Grupo de Trabajo de Atención Sanitaria en Residencias de Ancianos de la Sociedad Española de Geriatría y Gerontología. Prevalencia de demencia en pacientes institucionalizados: estudio RESYDEM. Rev Esp Geriatr Gerontol 2009; 44: 5-11.
3. Fratiglioni L, De Ronchi D, Aguero-Torres L. Worldwide prevalence and incidence of dementia. Drugs Aging 1999; 15: 365-75.

4. Canadian Study of Health and Ageing. Study methods and prevalence of dementia. CMAJ 1994; 150: 899-913.

5. Boller F, Verny M, Hugonot-Diener L, Saxton J. Clinical features and assessment of severe dementia: a review. Eur J Neurol 2002; 9: 125-36.

6. Mitchel SL, Teno JM, Kiely DK, Shaffer ML, Jones RN, Prigerson HG, et al. The clinical course of advanced dementia. N Engl J Med 2009; 361: 1529-38.

7. Buiza C. Evaluación y caracterización del deterioro cognitivo grave en personas con diagnóstico de enfermedad de Alzheimer [tesis doctoral]. Madrid: Universidad Autónoma de Madrid; 2011. URL: https://repositorio.uam.es/bitstream/handle/ 10486/6679/39532 buiza bueno_cristina.pdf.

8. Saxton J, McGonogle-Gibson KL, Swihart AA, Miler VJ, Boller F. Assessment of the severely impaired patient: description and validation of a new neuropsychological test battery. Psychol Assess 1990; 2: 298-303.

9. Llinàs-Regla J, Lozano M, López OL, Gudayol M, López-Pousa S, Vilalta J, et al. Validación de la adaptación española de la Severe Impairment Battery (SIB). Neurologia 1995; 10: 14-8.

10. Cruz-Orduña I, Agüera-Ortiz LF, Montorio-Cerrato I, LeónSalas B, Valle de Juan MC, Martínez-Martín P. Fiabilidad y validez de la batería de evaluación del deterioro grave, versión abreviada (SIB-s), en pacientes con demencia en España. Rev Neurol 2015; 60: 1-9.

11. Buiza C, Navarro A, Díaz-Orueta U, González MF, Álaba J, Arriola E, et al. Evaluación breve del estado cognitivo de la demencia en estadios avanzados: resultados preliminares de la validación española del Severe Mini-Mental State Examination. Rev Esp Geriatr Gerontol 2011; 46: 131-8.

12. Peavy GM, Salmon D, Rice V, Galasko D, Samuel W, Taylor K, et al. Neuropsychological assessment of severely demented elderly: the Severe Cognitive Impairment Profile. Arch Neurol 1996; 53: 367-77.

13. Mattis S. Mental status examination for organic mental syndrome in the elderly patient. In Bellak L, Karasu TB, eds. Geriatric psychiatry. New York: Grune \& Stratton; 1979. p. 77-121.

14. Folstein MF, Folstein SE, McHugh PR. Mini Mental State: a practical method for grading the cognitive state of patients for the clinician. J Psychiatr Res 1975; 12: 189-98.

15. Steinberg BA. Getting into dementia assessment on the ground floor.' Clin Neuropsychol 2001; 15: 235-9.

16. McKhann G, Drachman D, Folstein M, Katzman R, Price D, Stadlan EM. Clinical diagnosis of Alzheimer's disease: report of the NINCDS-ADRDA Work Group under the auspices of Department of Health and Human Services Task Force on Alzheimer's Disease. Neurology 1984; 34: 939-44.

17. Reisberg B, Ferris S, De Leon M, Crook T. The Global Deterioration Scale of assessment of primary degenerative dementia. Am J Psychiatry 1982; 139: 1136-9.

18. Lobo A, Ezquerra J. El 'mini examen cognoscitivo': un test sencillo, práctico, para detectar alteraciones intelectivas en pacientes psiquiátricos. Rev Psiquiatr Psicol Med 1980; 5 : 39-57.

19. Lobo A, Ezquerra J, Gomez-Burgada F, Sala JM, Seva-Díaz A El 'mini-examen cognoscitivo': un test sencillo, práctico, para detectar alteraciones intelectivas en pacientes médicos. Actas Luso Esp Neurol Psiquiatr 1979; 7: 189-202.

20. Albert MA, Cohen C. The Test for the Severe Impairment: an instrument for the assessment of patients with severe cognitive dysfunction. J Am Geriatr Soc 1992; 40: 449-53.

21. Doody RS, Strehlow SL, Massman PJ, Feher EP, Clark C, Roy JR. Baylor Profound Mental Status Examination: a brief staging measure for profoundly demented Alzheimer disease patients. Alzheimer Dis Assoc Disord 1999; 13: 53-9.

22. Monsch AU, Foldi NS, Ermini-Fünfschilling DE, Berres M, Taylor KI, Seifritz E, et al. Improving the diagnostic accuracy of the Mini-Mental State Examination. Acta Neurol Scand 1995; 92: 145-50. 
The validity and reliability of the Spanish version of the Severe Cognitive Impairment Profile (SCIP) for the cognitive assessment of persons with advanced stages of Alzheimer's disease

Introduction. Few validated tools exist in Spanish for conducting a precise assessment of the cognitive functioning of persons with advanced dementia. This population is a challenge due to the large number of persons who reach these states and owing to the complexity of the care that they require. This study presents a validated Spanish version of an instrument, the Severe Cognitive Impairment Profile (SCIP), which allows assessment of the cognitive functioning of persons with advanced Alzheimer's disease.

Patients and methods. We conducted a cross-sectional study with 133 subjects (29 males and 104 females; mean age: $81.61 \pm 7.41$ years) diagnosed with Alzheimer's disease in moderate to very severe stages, according to the Global Deterioration Scale (GDS 5-7). The following were performed: discrimination analyses of the items, a study of the internal consistency and interrater reliability and test-retest. Likewise, the factorial structure and the validity of the instrument were also tested.

Results. Only $1 \%$ of the 160 items on the scale were found to be non-discriminatory. The internal consistency of the scale is adequate, as is the interrater and the test-retest reliability. As regards the validity of the construct, the correlation with the mini-mental state examination is $0.74(p<0.01)$ and significant differences are found between the SCIP and the three levels of the GDS that were analysed. The factorial structure of the scales shows the existence of a single factor that accounts for $66.12 \%$ of the variance.

Conclusions. The SCIP scale is a useful, reliable and valid instrument for the exhaustive evaluation in Spanish of the cognitive functions of persons with dementia in moderate to very severe states.

Key words. Advanced dementia. Alzheimer's disease. Neuropsychological assessment. SCIP. Severe Cognitive Impairment Profile. Severe cognitive impairment. Validation. 